$R M x A C, \mathbf{5 3}, 100-107$ (2021)

(C) 2021: Instituto de Astronomía, Universidad Nacional Autónoma de México

https://doi.org/10.22201/ia.14052059p.2021.53.21

\title{
GAMMA-RAY BURSTS: A PERSONAL VIEW
}

\author{
G. J. Fishman ${ }^{1}$ \\ RESUMEN
}

Las primeras observaciones en astronomía de rayos gamma se realizaron a fines de la década de 1960, principalmente mediante observaciones desde globos estratosféricos. A principios de la década de 1970, los satélites descubrieron de manera fortuita unos estallidos de rayos gamma, cuando perseguían explosiones nucleares producidas por el hombre por encima de la superficie de nuestro planeta. La confirmación de estos estallidos gamma de origen cósmico pronto fue confirmada por otros satélites. El primer gran conjunto de detectores diseñado para observaciones de este nuevo fenómeno fue el instrumento BATSE en el Observatorio Compton de Rayos Gamma. Se describen algunos detalles de los instrumentos portados por globos y satélites así como dea las propiedades observacionales de estos estallidos de rayos gamma por ellos realizados.

\section{ABSTRACT}

The first observations in gamma-ray astronomy were made in the late 1960's, primarily by balloon-borne observations. In the early 1970's, gamma-ray bursts were discovered, completely by accident, by satellites looking for man-made nuclear explosions in space. The celestial nature of these events were soon confirmed by other satellites. The first large detector system designed for cosmic gamma-ray bursts observations was the BATSE instrument on the Compton Gamma-Ray Observatory. Some of the details of the instrumentation onboard ballons and satellites and the gamma-ray bursts observational properties they determined are presented.

Key Words: gamma-ray burst: general — space vehicles: instruments

\section{INTRODUCTION}

Various types of black-body radiation emitted by objects at different temperatures can be detected. For many centuries mankind could only see the heavens in visible light, a very small part of the radiation that is emitted by stars and galaxies in the sky.

In the late 1950's, X-ray detectors were sent on small sounding rockets above the Earth's atmosphere and this was the birth of X-ray astronomy. High-energy astronomy represents observations at the highest end of the electromagnetic spectrum: Xray astronomy and gamma-ray astronomy (Fig. 1).

\section{EARLY-TIME INSTRUMENTATION}

It was not until the space age in the 1960's, when sounding rockets and high altitude balloons, shown in Fig. 2, began to observe the sky. This was the beginning of High-Energy Astronomy: Xray and gamma-ray astronomy. With high-energy astronomy, many new types of high-energy objects in the sky would be discovered (such as the pulsed radiation from the Crab Nebula pulsar, Fishman et al. (1969)). These objects emitted most of their radiation as X-rays and gamma-rays, and little (or no) radiation was observed at lower energies.

\footnotetext{
${ }^{1}$ Senior Astrophysicist (Ret.), NASA-Marshall Space Flight Center, Hunstville, USA.
}

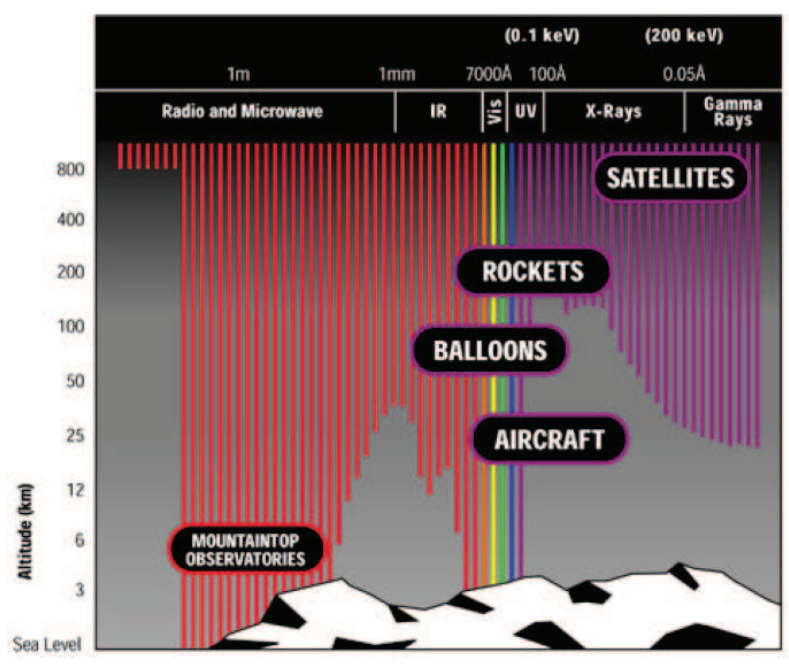

Fig. 1. The different classes of electromagnetic radiation and their different penetration into the atmosphere of the Earth.

In the 1970's and 1980's, the first large satellites were launched by NASA in the United States to study these $\mathrm{X}$-rays and gamma-rays. These were the three HEAO Spacecraft (see Fig. 3). It was with these three spacecraft that I started my professional career in high-energy astronomy, also coordinating the Guest Investigator programme (Fishman 


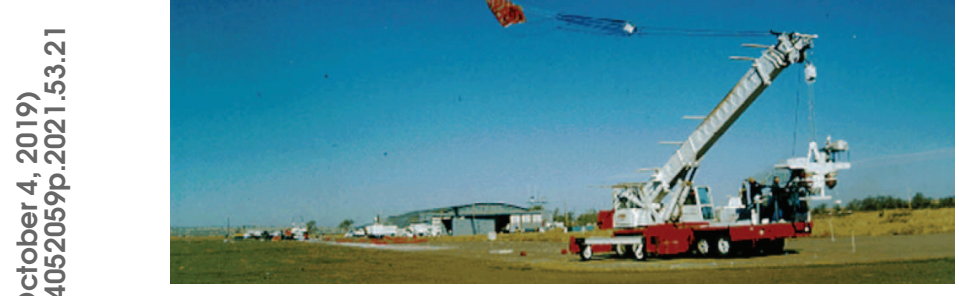

Fig. 2. Stratospheric globe carrying scientific instrumentation in its gondola before the launch.

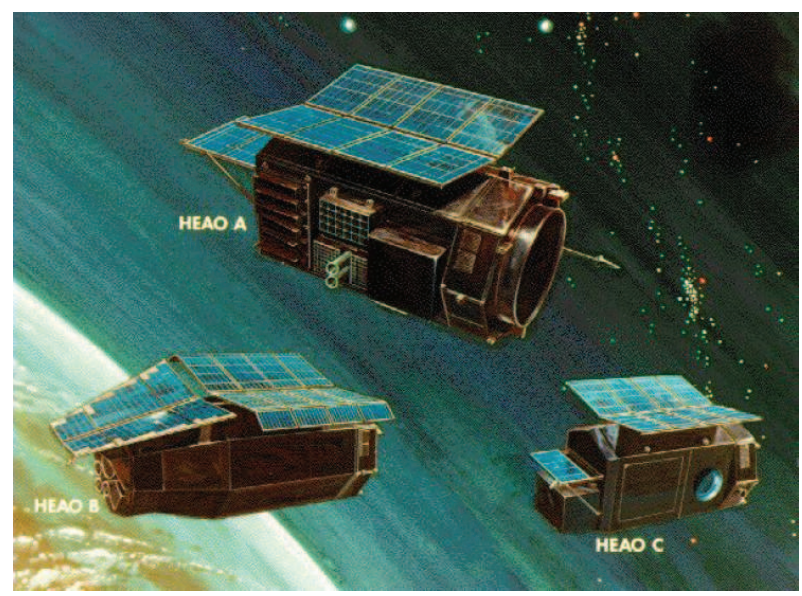

Fig. 3. The $H E A O$ scientific satellites.

et al. 1977). My own career started working with engineers on the development of these three satellites that were funded and managed by our NASA facility in Huntsville, Alabama, USA. These three HEAO spacecraft, launched in the late 1970's, carried the first very large instruments into space to observe Xrays, gamma-rays and cosmic rays from distant objects in our Galaxy (the Milky Way) and also from more distant galaxies in the Universe. These spacecraft were designed to make comprehensive studies of the newly discovered high-energy objects such as neutron stars and black holes. Neutron stars have the density of pure nuclear matter (without electrons) they have about the same density as if our entire Sun were squeezed into an area about the size of Málaga (the town in South Spain where the AstroRob 2019 Conference was hosted). These neutron stars emit most of their energy as X-rays and gamma-rays. Black holes are even more dense than
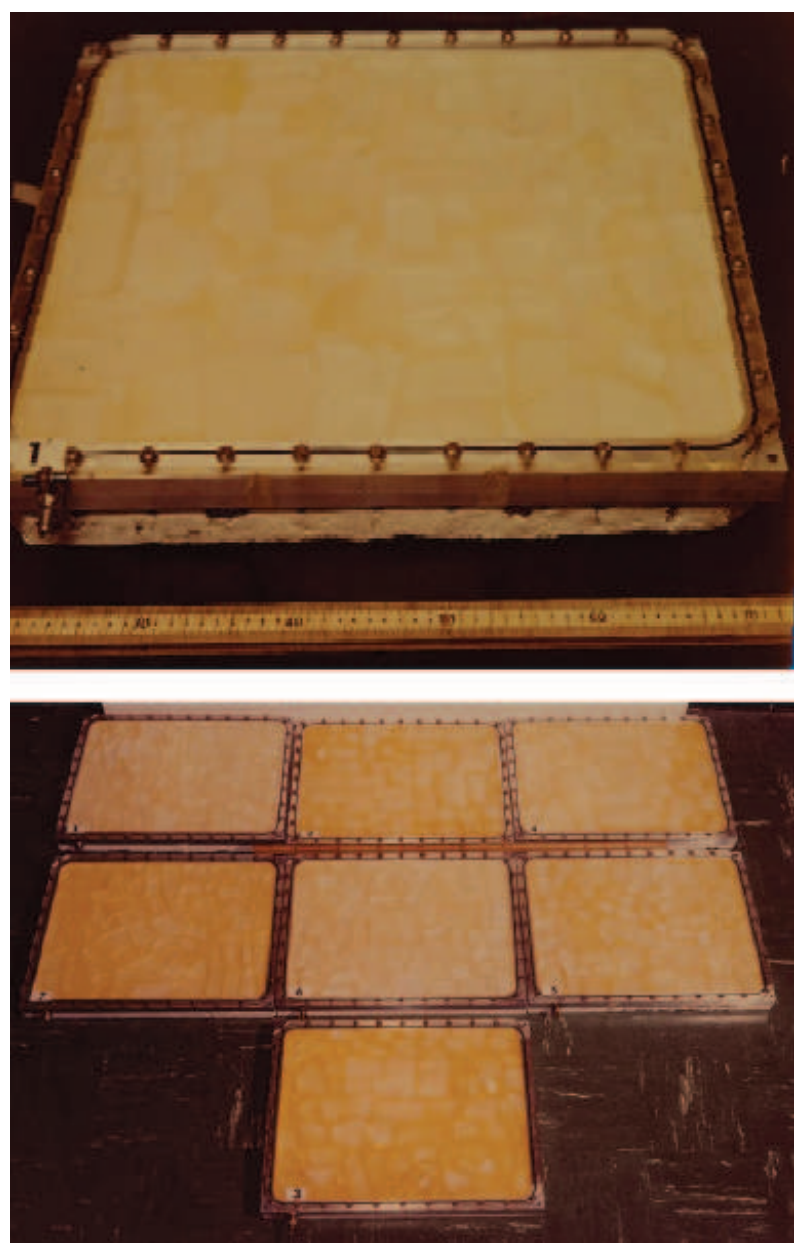

Fig. 4. Using large-area detectors made from NaI (Tl) crystal scrap pieces.

neutron stars. The gravity of black holes is so large, that nothing can escape, not even light. But just before matter enters the black hole, this material is heated to extremely high temperatures, so high that it emits large amounts of X-rays and gamma-rays. Many of these black hole objects have been detected by spacecraft in orbit around the Earth.

\section{THE WAY LEADING TO THE BATSE EXPERIMENT}

I conceived a large-area gamma-ray burst detector in the early 1970's in order to detect cosmic sources (such as the events previously recorded in the Vela satellites). We found a method of constructing a large volume, high efficiency scintillation detectors by immersing $\mathrm{NaI}(\mathrm{Tl})$ crystals in an optical coupling fluid. A large annulus was constructed by this method and used in a low-level counting system. The resolution of the annulus was found to be $45 \%$ at $661 \mathrm{keV}$, and its efficiency $\sim 50 \%$ at $1 \mathrm{MeV}$ (Fish- 

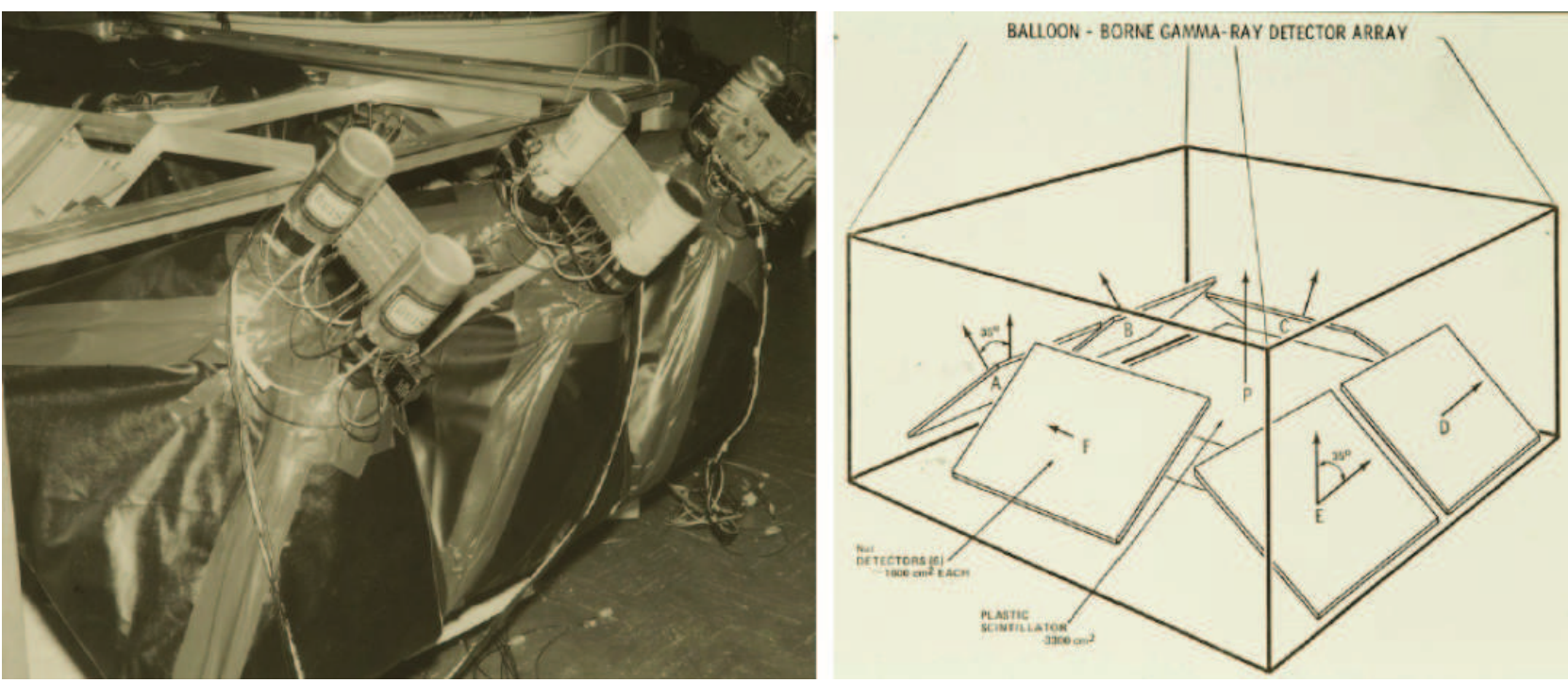

Fig. 5. Early balloon-borne gondola for detecting and providing rough localization from gamma-ray sources in 1976, with the detectors viewing different directions.

man and Walker 1973). The next step was using six large-area detectors made from $\mathrm{NaI}(\mathrm{Tl})$ crystal scrap pieces $\left(1500 \mathrm{~cm}^{2}\right)$ which were placed in a gondola and launched in a balloon flight in 1975 (Fishman 1981). This was the starting point of the NASA Marshall space flight center program in gamma-ray burst Astronomy.

The first flight in 1975 lasted $23 \mathrm{~h}$, but did not detect any gamma-ray burst. Instead, a significant geomagnetic north-south anysotropy in the background was observed, that could be due to small-angle scattering of electrons and high-energy gamma-rays in the residual atmosphere. Furthermore phosphorescent bursts were made with high time resolution, events that occur in crystal scintillator and are probably due to a high-Z cosmic ray or a local cosmic-ray shower, with the very large ones occurring at a rate of $1 \mathrm{~m}^{-2} \mathrm{~h}^{-1}$. See Fig. 4 .

The second flight in 1977 included six circular discs with six multicrystal detectors (reconditioned ones which were used for the first flight), with two photomultiplier tubes viewing each detector. During the $12 \mathrm{~h}$ flight, observation of discrete X-ray sources provided indication that the detectors had worked properly. See Fig. 5.

These ballon flights were launched from Palestine, Texas (USA), and floated at altitudes equivalent to 3-4 $\mathrm{gm} \mathrm{cm}^{-2}$ of residual atmosphere.

These flights were a very successful proof-ofconcept that paved the way for the Burst and Transient Source Experiment (BATSE) for the Gammaray Observatory. I proposed BATSE in 1979 and was selected in 1981. BATSE was the first large experiment designed for gamma-ray burst studies with two main advantages: full-sky coverage and use of large area detectors for improving the sensitivity.

In the mean time, we built a BATSE prototype by means of 20-inches diameter CsI detectors with a $2025 \mathrm{~cm}^{2}$ collecting area per single unit. Four such detectors were prepared for a third balloon flight from Palestine in 1979 which was also very successful. These detectors were similar to the proposed design for GRO, having photomultiplier tubes and improved light collection. A microprocessor-based data system was utilized which accomulated several different formats of spectral and temporal data. See Fig. 6.

This paved the way for BATSE and eight BATSE detectors were designed, fabricated, tested and calibrated at NASA Marshall Space Flight Center in Huntsville between 1982 and 1988 (see Fig. 7).

BATSE consisted of eight modules, one at each corner of the GRO spacecraft. In each module two detectors were placed: a Large Area Detector (LAD) and a Spectroscopy Detector (SD). The electronics were distributed between the detector modules and a unit within the spacecraft.

The LADs primary function was the detection and localization of bursts. The detectors were built around a thin $(1.27 \mathrm{~cm}$ wide) $\mathrm{NaI}(\mathrm{Tl})$ detector with an area of $2025 \mathrm{~cm}^{2}$. Only the front surface was shielded actively while passive shielding was utilized on the sides and back. The LADs operating in the 40-600 keV band. 

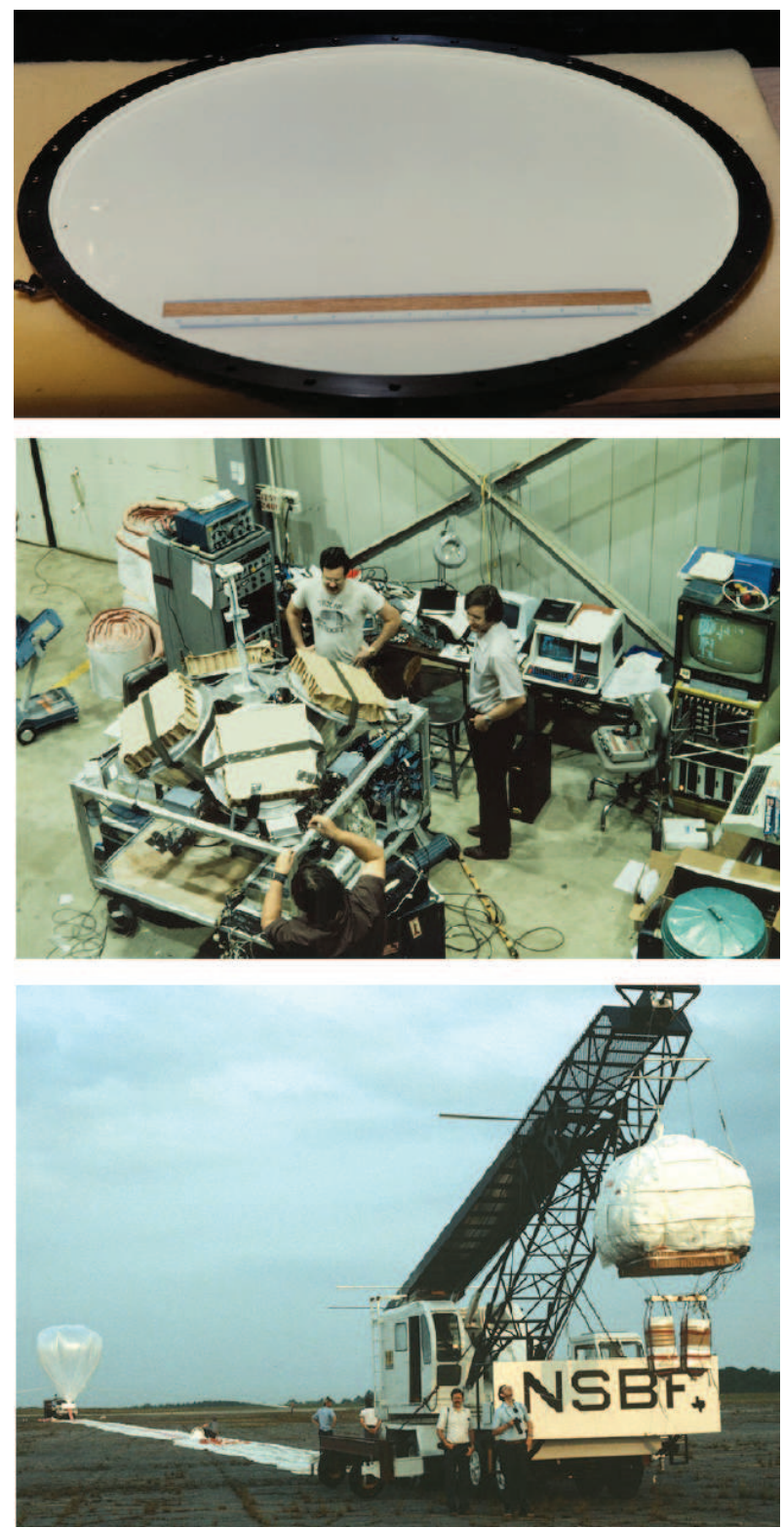

Fig. 6. BATSE-type detectors and an Early balloonborne gondola carrying four gamma-ray burst detectors, with the detectors viewing different directions.

SDs were optimized for accumulating a series of spectra across a burst with good temporal resolution. Each detector consisted of a thick $\mathrm{NaI}(\mathrm{Tl})$ crystal monitored by a single photomultiplier tube. The crystal had an area of $127 \mathrm{~cm}^{2}$ and a height of $7.6 \mathrm{~cm}$. The detectors were only shielded passively by the aluminum case with a beryllium window on the front surface. Depending on the photomultiplier gain, the SDs accumulated spectra between $15 \mathrm{keV}$ and $20 \mathrm{MeV}$. The SDs operated in the range 5-1500 $\mathrm{keV}$.
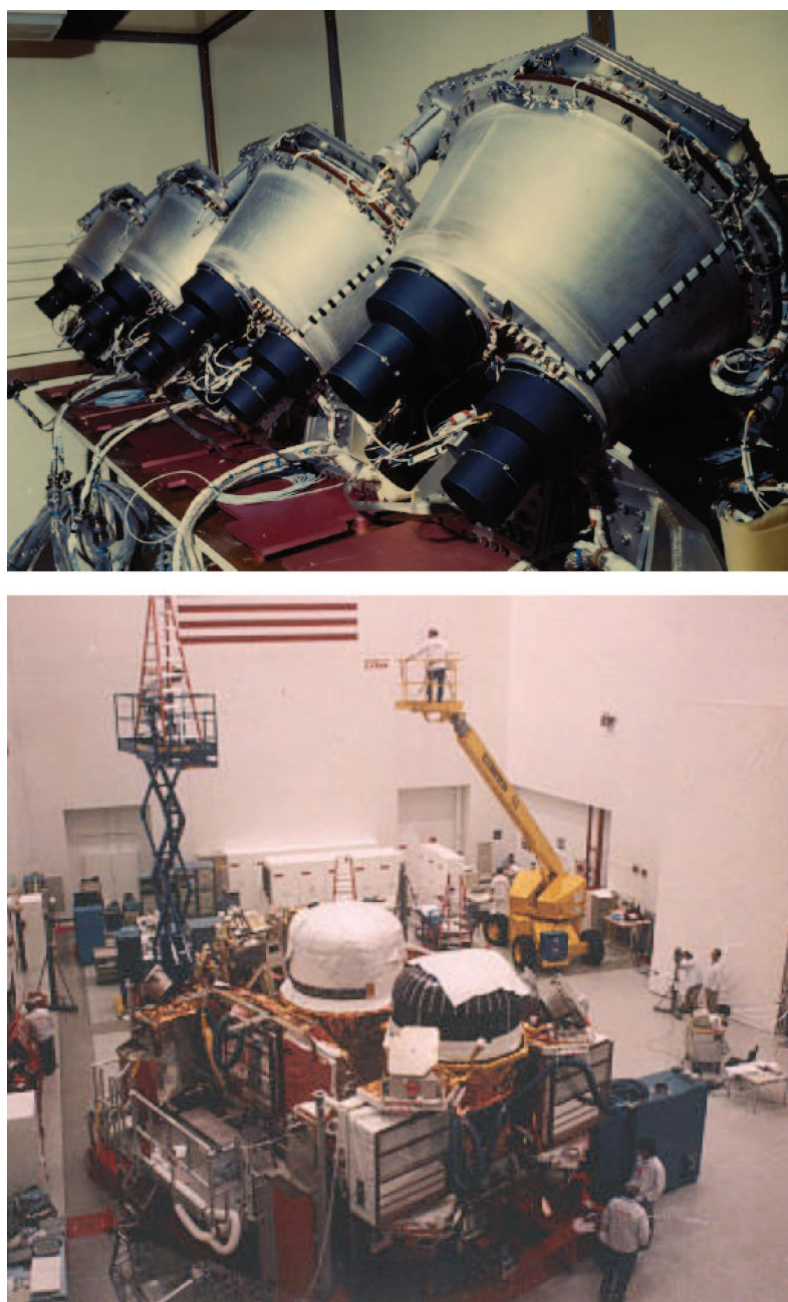

Fig. 7. BATSE detectors at NASA center in Huntsville and at the integration room together with the rest of instruments being attached to the Gamma-Ray Observatory spacecraft.

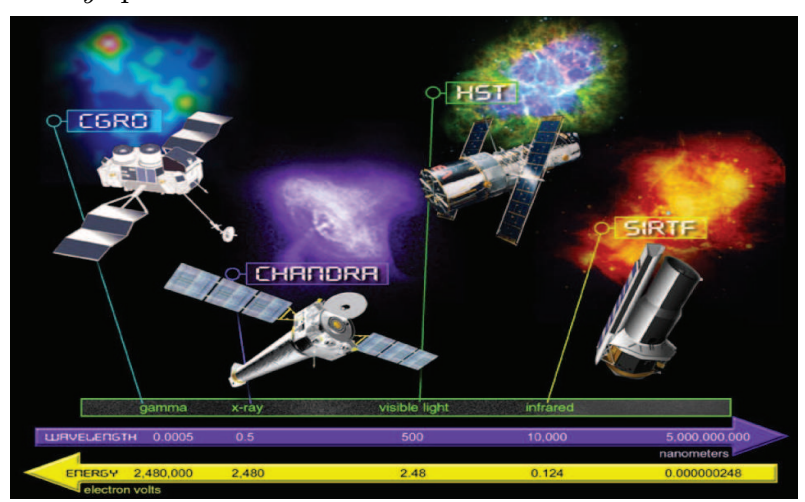

Fig. 8. The 4 scientific satellites of the NASA Great Observatories series launched into space in the 1990's. The Hubble Space Telescope (HST) is the best known of the four. 

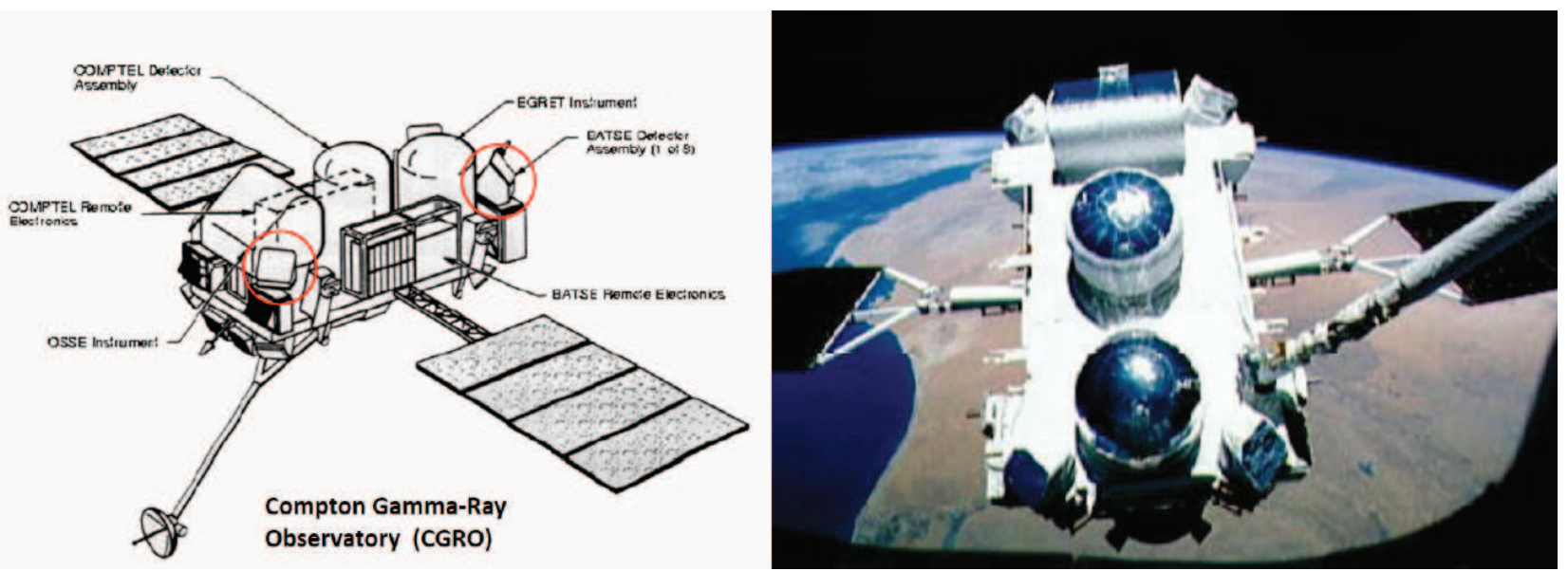

Fig. 9. The Compton Gamma-ray Observatory (CGRO) launched into space in 1991 from the space shuttle Atlantis.

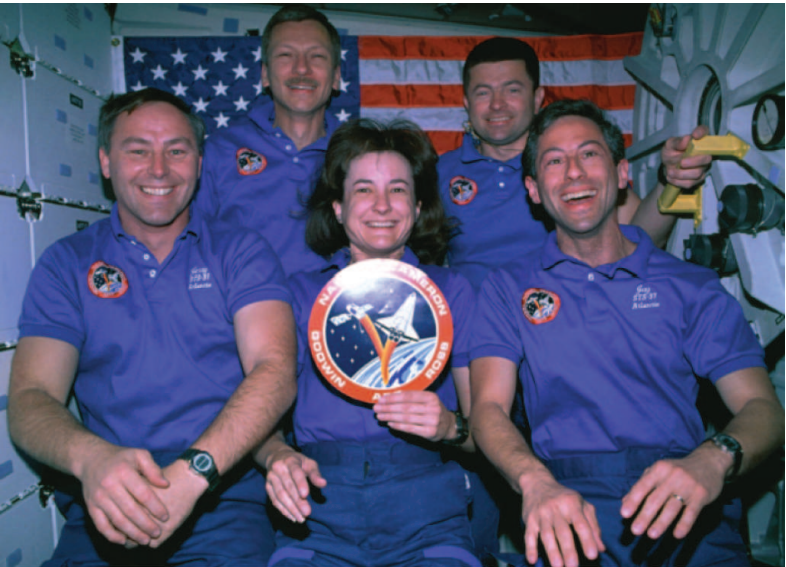

Fig. 10. The crew of the Space Shuttle Atlantis astronauts in charge of putting the CGRO into orbit. This photograph was taken by a camera onboard, just after the Compton Gamma-ray Observatory was safely placed into Earth orbit.

In the 1990's four much larger, massive spacecraft were launched by NASA. These were called "The Great Observatories in Space" (Fig. 8). Two of these massive spacecraft were launched by the U.S. Space Shuttle to observe objects that emit most of their radiation in the x-ray and gamma-ray regions of the electromagnetic spectrum. These are the Compton Gamma-ray Observatory and the Chandra X-ray Observatory. The majority of my career in high-energy astronomy was spent in making observations with the Compton Gamma-ray Observatory. See Fig. 9. Scientists around the world have used data from observations by this spacecraft.

This Gamma-Ray Observatory was launched into space and placed into orbit in 1991 by the U. S. Space Shuttle Atlantis. An instrument that I was in charge
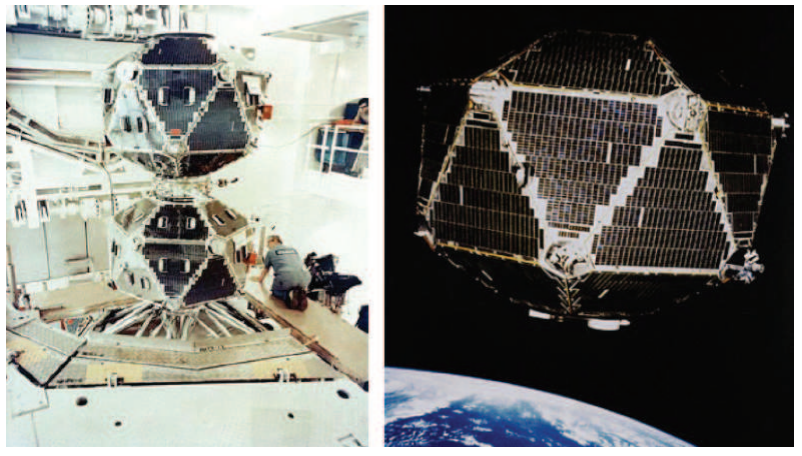

Fig. 11. The first gamma-ray bursts were discovered in 1973 by the Vela series spacecraft, designed for the detection of nuclear explosions in space.

of, and scientists have used data from, consisted of eight large detectors. These were placed at the eight corners of this spacecraft. The astronaut crew of Atlantis that carried this Compton Gamma-ray Observatory into space is shown in Fig. 10. The lady astronaut (and a friend of mine), Dr. Linda Godwin is holding up for the camera the mission insignia showing the names of the five Shuttle crew members. Over a period of nine years in orbit, the Compton Gamma-ray Observatory has made many discoveries regarding the nature of high-energy objects that emit gamma rays.

\section{GAMMA-RAY BURSTS}

The most explosive objects observed in the Universe are the Gamma-ray Bursts. These were discovered completely by accident in the early 1970's (Klebesadel et al. 1973). The United States had several nuclear bomb-detection satellites placed in very high orbits during the 1960's. These satellites were called the Vela satellites (Fig. 11). The U.S. was 

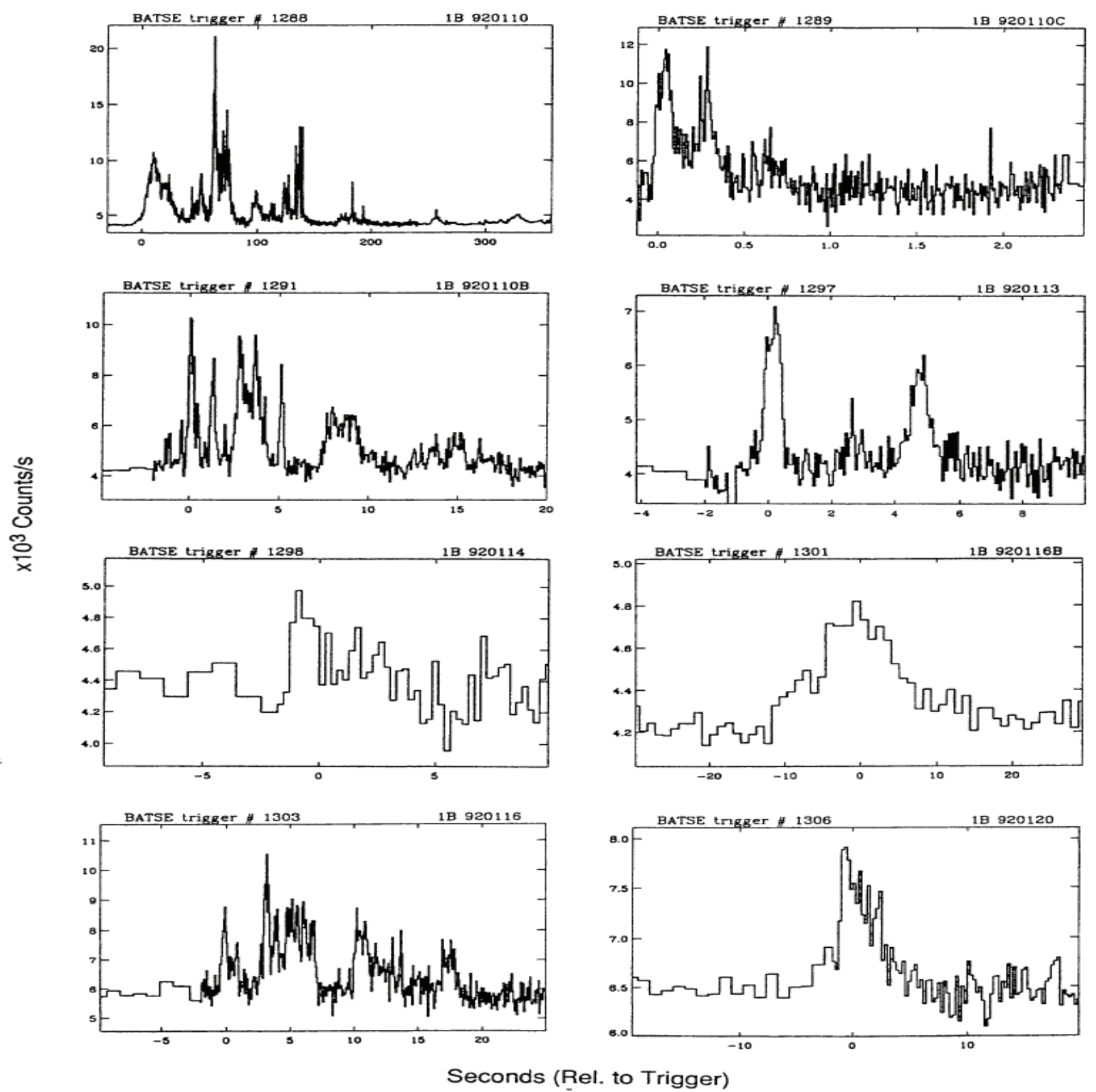

Fig. 12. A sample from the First BATSE Catalog of Gamma-Ray Bursts indicating the diversity in the time profiles, intensities and duration of GRBs. Adapted from Fishman and Meegan (1995).

worried that Soviet Union would try to perform some nuclear tests deep in outer space and they would try to hide these tests from the U.S. No such nuclear explosions were found, but every month or so, a large blast of gamma rays was seen by these small satellites. They appeared to be coming from outside of the Solar System, from some unknown objects. These unknown explosions were called "gamma-ray bursts" (GRBs). They occurred at random, and they were not coming from any known astronomical objects. It is these objects (gamma-ray bursts), that I have spent the majority of our careers observing and studying.

Probably the most striking features of the time profiles of the events were their morphological diversity and the large range of their durations. Examples of extreme morphologies and durations are showing in a sample of bursts detected by BATSE depicted in Fig. 12. See Fishman and Meegan (1995) for a review.

During their appearance, they often outshine all other sources in the gamma-ray sky combined. The 


\section{BATSE Gamma-Ray Bursts}

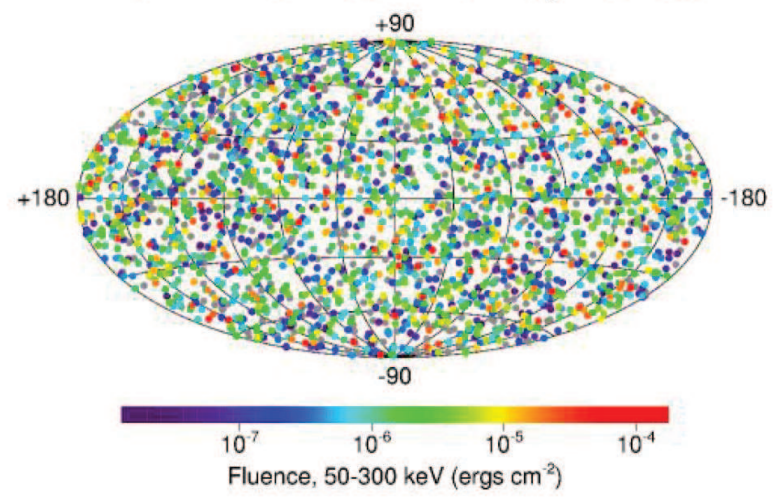

Fig. 13. Distribution in the celestial sphere of the 2704 gamma-ray bursts detected by the BATSE experiment aboard $C G R O$ during its operational life in space. From Meegan et al. (1992).

origin of these GRBs remained one of the biggest mysteries in astronomy for over two decades. There were dozens of theories that were suggested to explain the possible source of these blasts of gamma radiation. It was not even known whether they originated from within our own Milky Way galaxy, or from outside our galaxy, in a very distant galaxy.

It was not until many years later, when several thousand of these GRBs were located, that it became evident that GRBs were coming from random, distant locations of the sky, and not from our own Milky Way galaxy. It was also apparent that they originated from the most regions of the Universe. They represented the most powerful explosions known in the Universe.

Thanks to BATSE large detector system on board CGRO two major contributions to the GRB field were achieved:

- BATSE detected bursts at the rate of $\sim 1$ /day. The number versus intensity distribution did not follow the $-3 / 2$ power law expected for a spatially extended homogeneous distribution of sources, but at the same time the angular distribution was found to be isotropic within statistical limits. Taken together, these results were inconsistent with the spatial distribution of any known population of galactic objects, but was found to be consistent with the bursts being at cosmological distances (Meegan et al. 1992). See Fig. 13.

- A bimodality in the duration distribution of GRBs was observed, which separates GRBs into two classes: short events $(<2 \mathrm{~s})$ and longer ones

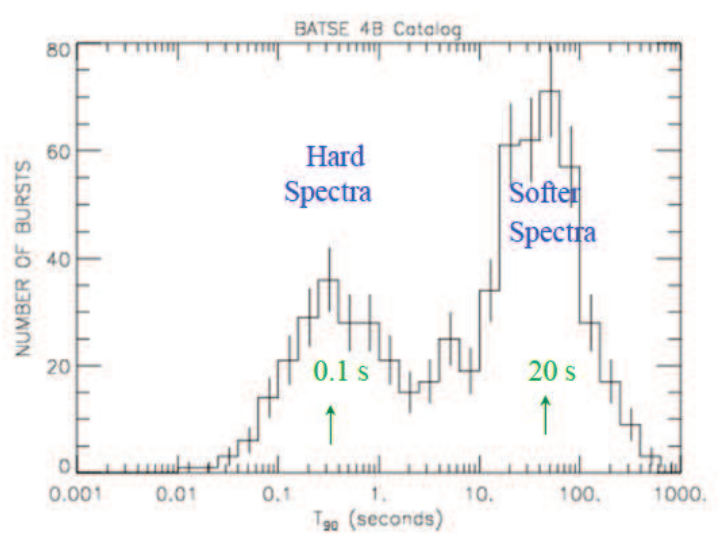

Fig. 14. Bimodal distribution of gamma-ray bursts detected by the BATSE experiment aboard CGRO during its operational life in space, showing the short-duration and the long-duration events. From Kouveliotou et al. (1993).

( $\geq 2 \mathrm{~s}$ ). Both sets were distributed isotropically and inhomogeneously in the sky. We found that their durations were anticorrelated with their spectral hardness ratios: short GRBs were predominantly harder, and longer ones tended to be softer. These results provided a first GRB classification scheme based on a combination of the GRB temporal and spectral properties, with the short-duration GRB population being $\sim 25 \%$ of the total number of events (Kouveliotou et al. 1993). See Fig. 14.

In addition to these findings in the GRB field, a major surprise provided by BATSE was the discovery of intense gamma-ray flashes of atmospheric origin (dubbed Terrestrial Gamma-ray Flases, TGFs). The photon spectra from the events were very hard (peaking in the high-energy portion of the spectrum) and were consistent with bremsstrahlung emission from energetic electrons (Fishman 1994). Today, it is estimated that $~ 500$ TGFs occur daily worldwide, but most go undetected. TGFs have been recorded to last 0.2 to $3.5 \mathrm{~ms}$, and have energies of up to $2 \times 10^{7} \mathrm{eV}$. TGFs pose a challenge to current theories of lightning. Among the processes of lightning there are mechanisms capable of generating gammarays, which escape the atmosphere and are observed by orbiting spacecraft.

\section{CONCLUSION}

The major part of my scientific career has been devoted to the study of GRBs. The experiments 
onboard balloons and the experience I gained in HEAO-3 paved the way to the BATSE experiment onboard $C G R O$. The eight large-area detectors allowed full-sky coverage and were optimized to operate in the primary energy region of emission of most GRBs. BATSE provided detailed observations of the temporal and spectral characteristics of several thousand GRBs, and it was the first experiment to provide rapid notifications of the coarse location of many them. It also provided strong evidence for the cosmological distances to GRBs through the observation of the sky distribution and intensity distribution of numerous GRBs (Fishman et al. 2013). The large number of GRBs observed with the high-sensitivity BATSE detectors continues to provide a database of GRB spectral and temporal properties in the primary energy range of GRB emission that will likely not be exceeded for at least another decade from now.

\section{REFERENCES}

Fishman, G. J., Harnden, F. R., Jr., \& Haymes, R. C. 1969, ApJ, 156, 107

Fishman, G. J. \& Walker, D. M. 1973, NucIM, 107, 357

Fishman, G. J. 1977, IAUC, 3066, 4 1981, Ap\&SS, 75, 125

Fishman, G. J., Bhat, P. N., Mallozzi, R., et al. 1994, Sci, 264, 1313

Fishman, G. J. \& Meegan, C. A. 1995, ARA\&A, 33, 415

Fishman, G. J. 2013, EAS, 61, 5

Klebesadel, R. W, Strong, I. B., \& Olson, R. A. 1973, ApJ, 182, 85

Kouveliotou, C., Meegan, C. A., Fishman, G. J., et al. 1993, ApJ, 413, 101

Megan, C. A., Fishman, G. J., Wilson, R. N., et al. 1992, Natur, 355, 153 